\title{
Positive feedback between retinoic acid and 2-phospho-L-ascorbic acid trisodium salt during somatic cell reprogramming
}

\author{
Mengdan Zhang 1,2,3,4, Qian Li, $i^{1,5}$, Tingting Yang ${ }^{1,2,3,4}$, Fei Meng ${ }^{1,2,3,4}$, Xiaowei Lai ${ }^{1,2,3,4}$, Lining Liang ${ }^{1,2,3}$, \\ Changpeng Li, ${ }^{1,2,3}$, Hao Sun ${ }^{1,2,3,4}$, Jiaqi Sun ${ }^{2}$ and Hui Zheng ${ }^{1,2,3,4,6^{*}}$
}

\begin{abstract}
Retinoic acid (RA) and 2-phospho-L-ascorbic acid trisodium salt (AscPNa) promote the reprogramming of mouse embryonic fibroblasts to induced pluripotent stem cells. In the current studies, the lower abilities of RA and AscPNa to promote reprogramming in the presence of each other suggested that they may share downstream pathways at least partially. The hypothesis was further supported by the RNA-seq analysis which demonstrated a high-level overlap between RA-activated and AscPNa activated genes during reprogramming. In addition, RA upregulated Glut1/3, facilitated the membrane transportation of dehydroascorbic acid, the oxidized form of L-ascorbic acid, and subsequently maintained intracellular L-ascorbic acid at higher level and for longer time. On the other hand, AscPNa facilitated the mesenchymal-epithelial transition during reprogramming, downregulated key mesenchymal transcriptional factors like Zeb1 and Twist1, subsequently suppressed the expression of Cyp26a1/b1 which mediates the metabolism of RA, and sustained the intracellular level of RA. Furthermore, the different abilities of RA and AscPNa to induce mesenchymal-epithelial transition, pluripotency, and neuronal differentiation explain their complex contribution to reprogramming when used individually or in combination. Therefore, the current studies identified a positive feedback between RA and AscPNa, or possibility between vitamin A and C, and further explored their contributions to reprogramming.
\end{abstract}

Keywords: Reprogramming, L-ascorbic acid, Retinoic acid, Positive feedback, Mesenchymal-epithelial transition

\section{Background}

In 2006, Yamanaka and colleagues introduced four transcription factors into mouse embryonic fibroblasts (MEFs) and successfully generated induced pluripotent stem cells (iPSCs) (Takahashi and Yamanaka 2006). Then, the abilities of small-molecular compounds, including vitamins, to regulate reprogramming have attracted great attention (Esteban et al. 2010; Wang et al. 2011a).

\footnotetext{
* Correspondence: zheng_hui@gibh.ac.cn

${ }^{1}$ CAS Key Laboratory of Regenerative Biology, Guangzhou Institutes of Biomedicine and Health, Chinese Academy of Sciences, \#190 Kaiyuan Ave., Science City, Guangzhou 510530, Huangpu District, China

${ }^{2}$ Bioland Laboratory (Guangzhou Regenerative Medicine and Health Guangdong Laboratory), Guangzhou 510700, China

Full list of author information is available at the end of the article
}

Vitamin C (Vc) promotes reprogramming and increases the quality of generated iPSCs (Esteban et al. 2010; Esteban and Pei 2012). Mechanisms involving Vcdependent H3K36 demethylases, Nanog and other pluripotency genes, and Ten-eleven translocation 1 (Tet1) have been reported (Wang et al. 2011b; Gao et al. 2013; Wu et al. 2014; Chen et al. 2013). Normally, 2-phospho$\mathrm{L}$-ascorbic acid trisodium salt (AscPNa) is used to provide $\mathrm{L}$-ascorbic acid (Asc) during reprogramming with the help from phosphatase (Austria et al. 1997; Kameyama et al. 1996). Sodium-dependent vitamin C transporters (SVCTs) transport Asc into cells and maintain its intracellular concentration (Linster and Van Schaftingen E. Vitamin C. 2007). L-dehydroascorbic acid 
(DHAA), the oxidized form of Asc, is transported into cells by glucose transporters (GLUTs) and maintains intracellular concentration of Asc after being reduced (Linster and Van Schaftingen E. Vitamin C. 2007). The oxidation-reduction between Asc and DHAA and these membrane transporters, SVCTs and GLUTs, are critical for maintaining the balance of Asc across cell membrane (Wilson 2005).,

Vitamin A (Va) is normally obtained as all-trans-retinol, retinyl esters or $\beta$-carotene from diet (Mora et al. 2008). Retinoic acid (RA) is synthesized from retinol and activates the transcription of target genes after binding to its nuclear receptors. RA signaling pathway is reported to facilitate somatic cell reprogramming (Wang et al. 2011a; Yang et al. 2015). In addition, Deng and his colleagues generate iPSCs using chemical-defined medium which contains AM580 (agonist of RA receptor $\alpha$ ) and TTNPB (a synthetic ligand of RA receptor) (Hou et al. 2013; Zhao et al. 2015; Masuda et al. 2013).

RA removes DNA methylation in embryonic stem cells (ESCs) by upregulating TET2 (Bar-El Dadon and Reifen 2017). In addition, both RA and AscPNa enhance the reprogramming to naïve pluripotency by modulating the levels and activities of TET proteins (Hore et al. 2016). Mesenchymal-epithelial transition (MET) is considered to be an essential process during early stage of fibroblast reprogramming (Li et al. 2010; Shu and Pei 2014). AscPNa promotes MET and facilitates reprogramming of MEFs (Chen et al. 2013). As a MET inducer, Klf4 is also a downstream target of RA pathway (Li et al. 2017; Shi et al. 2012). Besides, RA is used as a potential chemo-therapeutic agent because of its important role in inhibiting epithelial-mesenchymal transition (EMT) (Cui et al. 2016; Guan et al. 2014). Thus, both RA and AscPNa regulate TET-mediated DNA demethylation and MET.

Therefore, an interaction between the downstream pathways of these two vitamins is possible, and the current studies were performed to confirm this hypothesis.

\section{Results}

\section{AscPNa and RA impair the abilities of each other to promote reprogramming}

To studies the functions of AscPNa and RA during reprogramming, three different kinds of media were used during reprogramming, including conventional $\mathrm{mES}$ medium, E8 medium, and N2B27 medium. mES medium contains approximately $15 \%$ fetal bovine serum (FBS) and is a traditional medium used for reprogramming (Esteban et al. 2010). The current E8 medium was modified from a previously reported chemical-defined medium, but without AscPNa, TGF $\beta$ or Nodal (Chen et al. 2011). N2B27 medium has been used to study the function of RA during reprogramming (Wang et al. 2011a), and B27 supplement without RA was used instead in the current studies.

As indicated in Fig. 1a-d, the Oct4-GFP ${ }^{+}$colonies were counted on day 12 in the presence of AscPNa. Actually, no Oct4-GFP ${ }^{+}$colonies were identified on day 12 without AscPNa. Thus, AscPNa promoted reprogramming no matter what kind of medium was used (Fig. 1b-d).

The abilities of RA to promote reprogramming were concentration- and medium-dependent. Ten nM RA promoted reprogramming with N2B27 or E8 medium, while impaired reprogramming with $\mathrm{mES}$ medium (Fig. 1b-d). One $\mu \mathrm{M}$ RA had lower ability than $10 \mathrm{nM}$ RA to promote reprogramming with N2B27 medium, but had higher ability to impair reprogramming with mES medium (Fig. 1b-d). Thus, the inhibitory effects of RA increased along with the increase of RA concentration, while the beneficial effects decreased.

By controlling the time points (day 23-25) at which the Oct4-GFP ${ }^{+}$colonies were counted, the numbers of Oct4-GFP ${ }^{+}$colonies in "DMSO" groups during reprograming without AscPNa were similar to those in "DMSO" groups during reprograming with AscPNa (Fig. 1b-d). Although the amounts of Oct4-GFP ${ }^{+}$colonies were similar, the counting for reprogramming with AscPNa is much earlier (at least 11-day earlier) than that for reprogramming without AscPNa, which confirmed the abilities of AscPNa to promote reprogramming. In addition, when the amounts of Oct4-GFP ${ }^{+}$colonies were similar in "DMSO" groups during two kinds of reprogramming, it is easier to identify the potential interaction between RA and AscPNa.

As indicated with N2B27 medium in Fig. 1d, the abilities of $10 \mathrm{nM}$ or $1 \mu \mathrm{M}$ RA to promote reprogramming were significantly impaired in the presence of AscPNa. In addition, although the amounts of Oct4- $\mathrm{GFP}^{+}$colonies were similar in "DMSO" groups during two kinds of reprogramming, less Oct4-GFP ${ }^{+}$colonies were observed in the presence of AscPNa than in the absence of AscPNa in "10 nM" or " $1 \mu \mathrm{M}$ RA" groups (Fig. 1d). Consistent results were obtained with $\mathrm{mES}$ or E8 medium in Fig. 1b-c. Therefore, RA and AscPNa have lower abilities to promote reprogramming in the presence of each other.

\section{$\mathrm{RA}$ and AscPNa share downstream target genes}

There are several possible explanations for the observations in Fig. 1b-d. One is the cross-talk or overlap between the downstream pathways of RA and AscPNa. To confirm this, we performed RNA-seq on day 6 during the reprogramming with N2B27 medium. Top-500 upregulated and downregulated genes during reprogramming were selected based gene expression in MEFs and ESCs. The expression of these genes in the current 


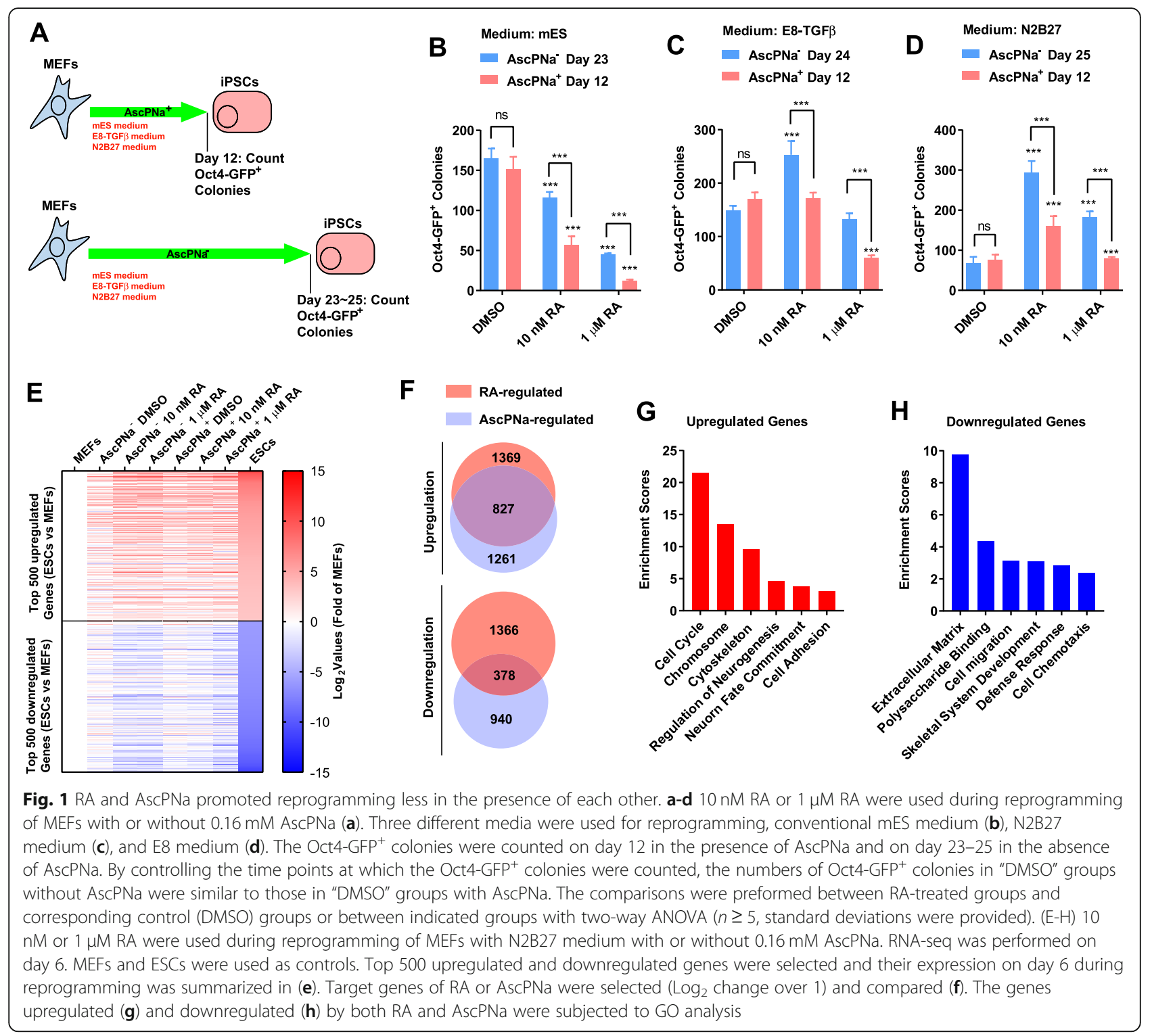

RNA-seq results were summarized in Fig. 1e. Larger modulations of these genes in the presence of RA or AscPNa confirmed the abilities of RA and AscPNa to promote reprogramming.

The genes regulated by RA or AscPNa were then selected and analyzed. RA and AscPNa significantly upregulated 1369 and 1261 genes respectively, and 827 of them were upregulated by both molecules (Fig. 1f). This high-level overlap suggested that RA and AscPNa might share downstream pathways. Gene Oncology (GO) analysis indicated that these overlapping genes were enriched in categories related to cell cycle, neurogenesis, and cell adhesion (Fig. 1g).

The genes downregulated by RA and AscPNa were less overlapped. RA and AscPNa significantly downregulated 1366 and 940 genes respectively, and only
378 genes were downregulated by both molecules (Fig. 1f). These genes were enriched in categories related to extracellular matrix, cell migration, and cell chemotaxis (Fig. 1h).

\section{RA enhances the transportation of DHAA}

One possible explanation for the overlap is that RA and AscPNa modulate the synthesis or degradation of each other. However, the genes which participate in the synthesis or degradation of Asc (based on Kyoto Encyclopedia of Genes and Genomes, KEGG, pathway 00053, ascorbate and aldarate metabolism) were not significantly modulated by RA during reprogramming (Table S1).

SVCTs and GLUTs which respectively transport Asc and DHAA into cells and maintain intracellular 
concentration of Asc (Linster and Van Schaftingen E. Vitamin C. 2007) were then analyzed. The expression of Glut2, Glut4, and Svct1 were not detected in the current RNA-seq (Table S1). In addition, RA significantly upregulated the membrane transporters of DHAA, Glut1 and Glut3 (also known as Slc2a1 and Slc2a3), but did not affect the expression of Svct2 (also known as Slc23a2) (Table S1). Increase of the protein levels of Glut1 and Glut3 were observed on day 6 during reprogramming (Fig. 2a). Pscan software identified binding site of RARA::RARG on the promoters of Glut1 and Glut3 (Zambelli et al. 2009; Meneses et al. 2008; Lu et al. 2020), which was confirmed when the activities of their promoters of (both wild type and RARA::RARG binding site mutation) were determined in the presence and absence of RA (Fig. 2b-c). RA treatment increased the activities of the wild type promoters of Glut1 and Glut3, but not the promoters with the binding sites of RARA:: RARG mutated (Fig. 2b-c).

During reprogramming with N2B27 medium containing AscPNa, the intracellular level of Asc was determined on day 6 (24 h after replacing the medium with fresh medium). RA increased the intracellular Asc in a concentration-dependent manner (Fig. 2d).

The intracellular level of Asc was also determined in MEFs in the presence of AscPNa or DHAA (in N2B27 medium) (Fig. 2e-j). Since DHAA is less stable than AscPNa or Asc (Wilson 2002), the intracellular level of Asc was monitored for $12 \mathrm{~h}$ in the presence of DHAA, while $24 \mathrm{~h}$ in the presence of AscPNa. During the treatment of DHAA or AscPNa, the intracellular Asc increased at the early stage and gradually decreased to basal level at the late stage (Fig. 2e-j). RA treatment accelerated the initial increase of intracellular Asc at the early stage and impaired the decrease of intracellular Asc at the late stage (Fig. 2e-j). Thus, RA helps to sustain the intracellular level of Asc. In addition, when used in MEFs, RA significantly upregulated the expression of Glut1 and Glut3 but not Svct2 (Fig. 2k-n). Therefore, RA maintains higher intracellular Asc by facilitating the DHAA transportation across cell membrane.

\section{AscPNa impairs the degradation of RA}

The ability of AscPNa to affect the intracellular RA was then determined. RA is normally degraded by CYP26A1 and CYP26B1 (Gottesman et al. 2001). The expression of these two genes was stimulated by RA in a concentration-dependent manner, which was suppressed at least partially in the presence of AscPNa (Fig. 3a-b).

Three RAREs and a mini-TK promoter were placed before Egfp reporter gene and used to determine the intracellular level of RA (Fig. 3c). The reporter was delivered into MEFs via a lentivirus system. MEF medium was replaced with N2B27 medium $12 \mathrm{~h}$ after an additional passage of MEFs. $10 \mathrm{nM}$ RA treatment increased the EGFP fluorescence and the peak level was reached after three-day treatment (Fig. 3d). When RA was withdrawn on day 3, the EGFP fluorescence decreased gradually. In addition, adding AscPNa at the same time with RA withdrawal prevented the decrease of EGFP fluorescence. Thus, AscPNa sustains the intracellular level of RA.

The promoters of Cyp26a1 and Cyp26b1 were also placed before the Egfp reporter gene (Fig. 3c). RA treatment and RA withdrawal initiated the increase and decrease of Egfp expression, respectively (Fig. 3e-f). However, adding AscPNa at the same time with RA withdrawal (day 3) facilitated the decrease of Egfp expression. Therefore, AscPNa maintains the intracellular level of RA by suppressing Cyp26a1/b1 and impairing RA degradation.

AscPNa treatment decreased the activities of Cyp26a1 and Cyp26b1 promoters, it is reasonable to suggest that several downstream transcriptional factors of AscPNa might bind to these two promoters. AscPNa-induced MET is a possible explanation. To confirm this, TGF $\beta$ and Repsox, a TGF pathway inhibitor, were then used to induce EMT and MET respectively in MEFs in the presence of $10 \mathrm{nM}$ RA in N2B27 medium. TGF $\beta$ activated while Repsox inhibited the promoters of Cyp26a1 and Cyp26b1 (Fig. 3g). In addition, TGF $\beta$ activated while Repsox inhibited the expression of Cyp26a1 and Cyp26b1 in MEFs (Fig. 3h).

Binding sites of key mesenchymal transcriptional markers, like ZEB1 and TWIST1 were identified on the promoters of Cyp26a1 and Cyp26b1 by Pscan software (Zambelli et al. 2009; Hu et al. 2008; El Hokayem et al. 2018) (Fig. 3i). In addition, overexpression of Zeb1 and Twist1 increased the activity of wild type promoters of Cyp26a1 and Cyp26b1 respectively, but not the promoters with the corresponding binding sites mutated. (Fig. 3j-k). Therefore, AscPNa suppresses the expression of Cyp26a1 and Cyp26b1 by inducing MET and suppressing these key mesenchymal transcriptional factors.

\section{RA has high ability to induce MET and pluripotency}

The genes which were significantly regulated by RA but not by AscPNa were selected and analyzed. These genes were enriched in the categories related to extracellular matrix and cell motion (Fig. S1A-B). The RNA-seq results indicated that RA had high ability to induce MET (Fig. S1C).

As a MET inducer, Klf4 can be upregulated by RArelated pathway (Li et al. 2017; Shi et al. 2012). To determine whether other key transcriptional factors in ESCs were also regulated by RA, top 500 upregulated genes during reprogramming were analyzed. Among these genes, Nanog and Dppa5a attracted great attention. To 


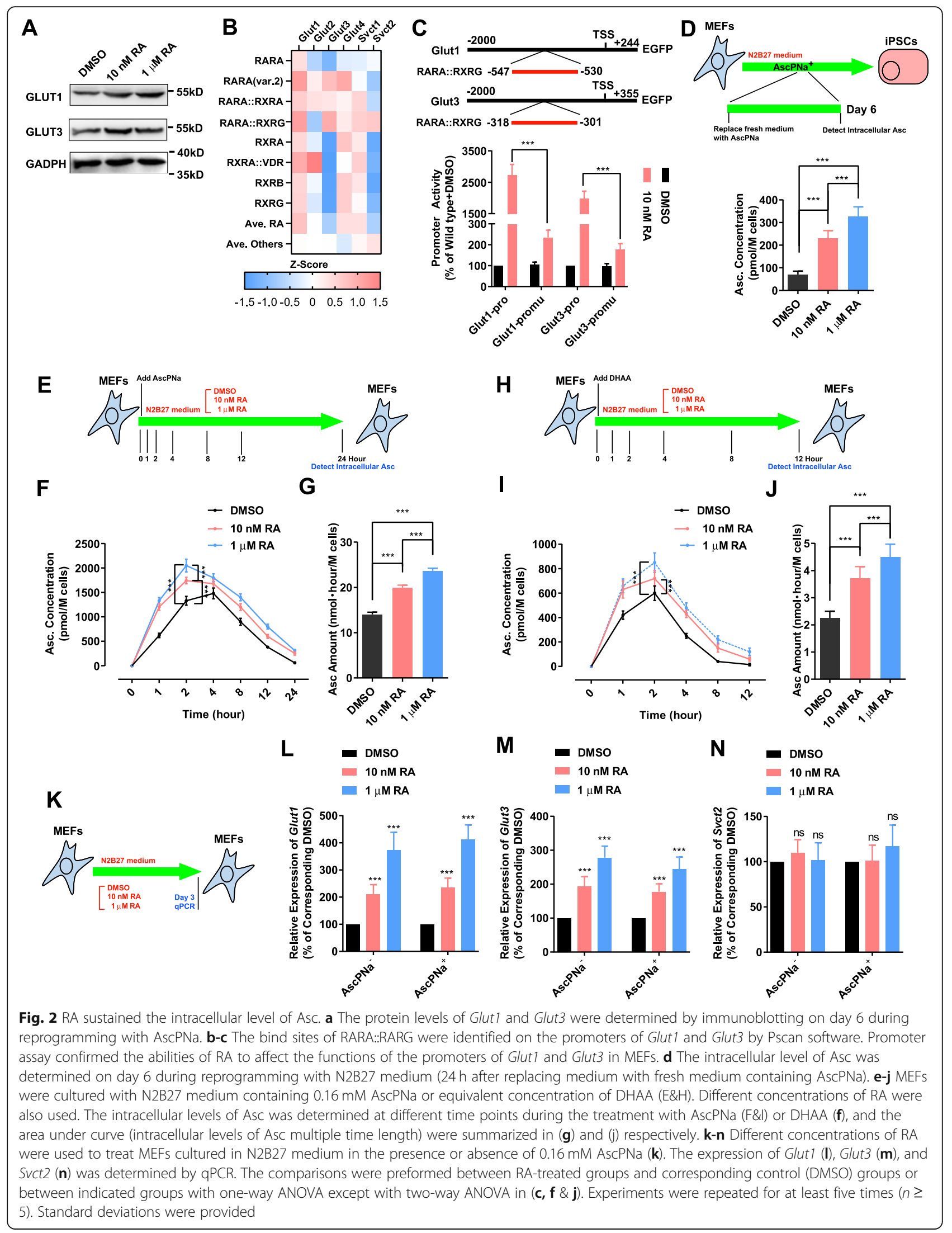




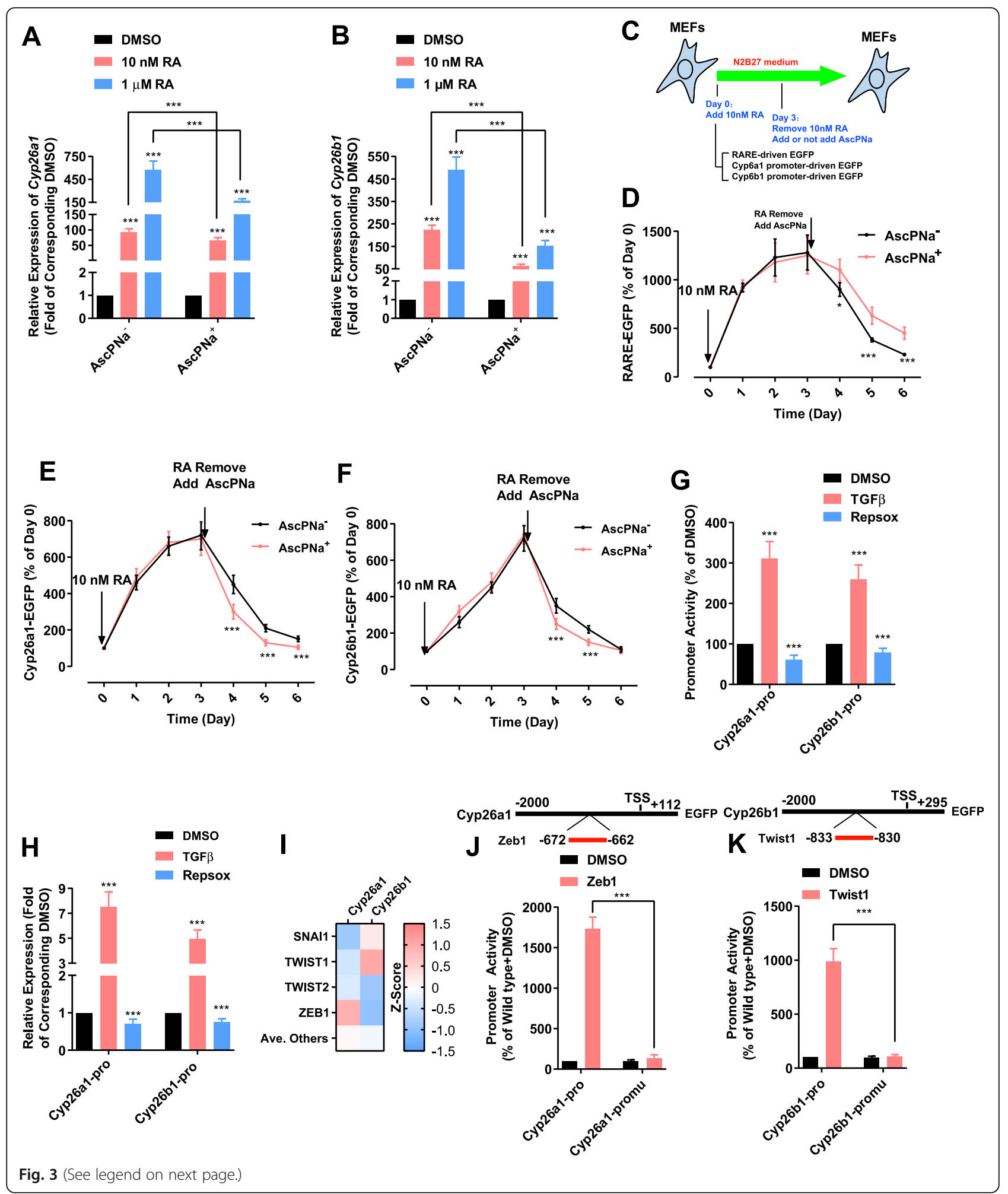


(See figure on previous page.)

Fig. 3 AscPNa impaired the degradation of RA. a-b Different concentrations of RA were used during reprogramming of MEFs with N2B27 medium in the presence or absence of $0.16 \mathrm{mM}$ AscPNa. The expression of Cyp26al (a) and Cyp26b1 (b) was determined by qPCR. c-f EGFP reporter genes driven by different promoters were delivered to MEFs via lentivirus system (c). N2B27 medium was used $24 \mathrm{~h}$ after the delivery. 10 nM RA was included in the medium for the next three days. After the removal of RA, 0.16 mM AscPNa and PBS were used in two different groups. EGFP fluorescence was determined at indicated time points. RARE-containing mini promoter was used to indicate RA concentration in (d). Promoters of Cyp26a1 (e) and Cyp26b1 (f) were also used. g-h MEFs were cultured in N2B27 medium. Different concentrations of RA were used for three days. The activities of the promoters of Cyp26a1 and Cyp26b1 (g) and the expression of Cyp26a1 and Cyp26b1 (h) were determined by FACS and APCR, respectively. i-k The bind sites of ZEB1 and TWIST1 were identified on the promoters of Cyp26a1 and Cyp26b1 by Pscan software, respectively. Promoter assay confirmed the abilities of ZEB1 and TWIST1 to affect the functions of the promoters of Cyp26a1 and Cyp26b1. The comparisons were preformed between RA-treated groups and corresponding control (DMSO) groups or between indicated groups with one-way ANOVA except in (d-f). The comparisons in ( $\mathbf{d}-\mathbf{f}, \mathbf{j}-\mathbf{k}$ ) were preformed between $\mathrm{AscPNa}^{+}$and $\mathrm{AscPNa}^{-}$groups or between pro or promu with two-way ANOVA. Experiments were repeated for at least five times $(n \geq 5)$. Standard deviations were provided

confirm the ability of RA to regulate the expression of these genes, RA was used to treat MEFs cultured in N2B27 medium for three days. RA increased the expression of Klf4, Nanog, and Dppa5a in a concentrationdependent manner (Fig. S1D-F).

\section{RA induces neuronal differentiation of PSCs}

RA is reported to promote the neuronal differentiation of PSCs (Gudas and Wagner 2011). Thus, high concentration of RA may induce differentiation of iPSCs generated during reprogramming, and subsequently result in a lower reprogramming efficiency. Treatment with $1 \mu \mathrm{M}$ RA preferentially upregulated genes related to embryonic morphogenesis, forebrain development and retinol metabolism (Fig. S2A-B).

To further confirm the abovementioned hypothesis, the expression of neuronal markers was analyzed in
Table S1. The expression of three neural lineage markers, Nes, Prom 1 and Ascl1, were identified to be upregulated by RA during reprogramming (Table S1). When RA was used to treat MEFs cultured in N2B27 medium for three days, it increased the expression of Nes, Prom 1 and Ascl1 in a concentration-dependent manner (Fig. S2C-E).

\section{Conclusions}

In the current studies, positive feedback was identified between RA and AscPNa, during somatic reprogramming (Fig. 4). RA upregulates the expression of the membrane transporters of DHAA, Glut1 and Glut3, maintains the intracellular level of Asc, and subsequently promotes reprogramming. AscPNa induces MET, suppresses the expression key mesenchymal transcriptional

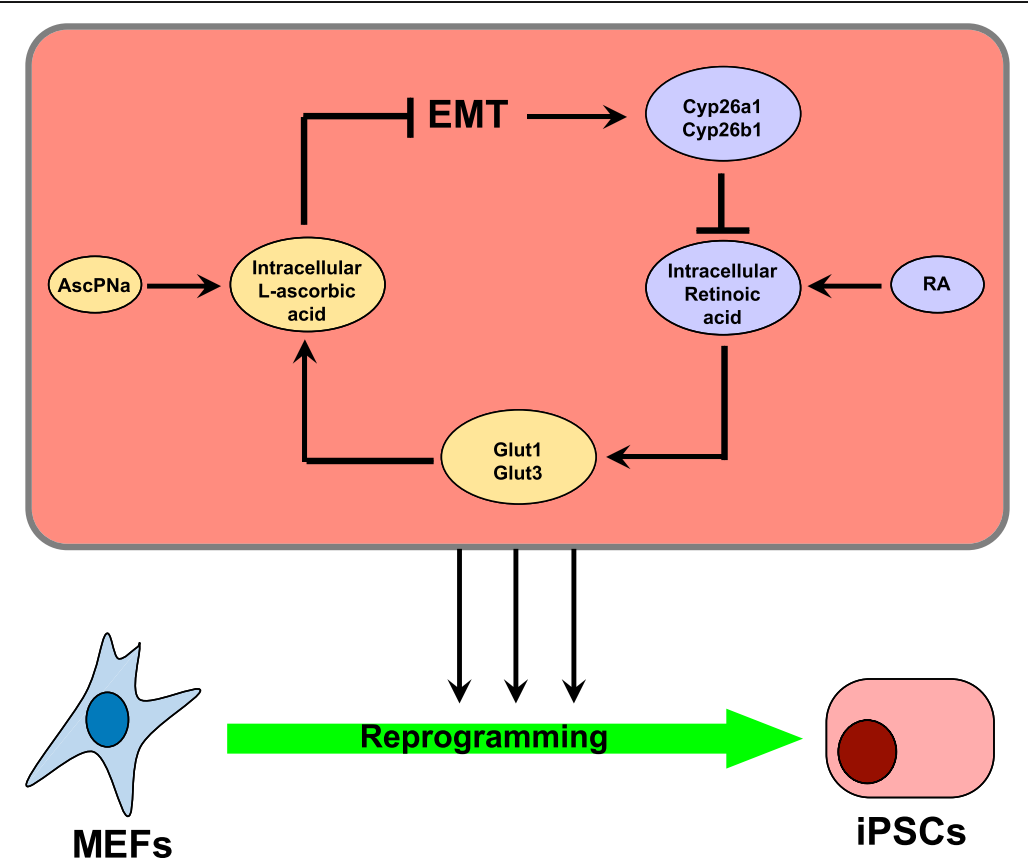

Fig. 4 Schematic summary of the positive feedback between Va and Vc 
factors, resulting in the downregulation of Cyp26a1 and Cyp26b1, and impairs the degradation of RA.

Although RA and AscPNa were not provided in N2B27 medium (B27 used in the current studies does not contain RA), the two vitamins were not eliminated totally during the current reprogramming. The retrovirus used to deliver four Yamanaka factors into MEFs was packaged in the Plat-E cells cultured in MEF medium (Table S1 and Materials and Methods). Since FBS is an important component of MEF medium, Va and $\mathrm{Vc}$ were provided to the cells via FBS during virus infection. The remaining $\mathrm{Va}$ and $\mathrm{Vc}$ in the cytosol of MEFs after changing MEF medium to N2B27 medium during reprogramming also prevented us to eliminate these vitamins totally.

When RA was used alone during the reprogramming with N2B27 medium, little Asc or DHAA remained in the medium. Thus, upregulation of Glut1 and Glut3 might not be able to sustain intracellular Asc. However, as reviewed by previous reports (Linster and Van Schaftingen E. Vitamin C. 2007; Wilson 2002), there are also mechanisms allowing the efflux of Asc or DHAA from cells. Thus, the membrane transporters for Asc or DHAA also function to maintain the balance between extracellular and intracellular Asc. Upregulation of Glut1 and Glut3 shifts the balance towards intracellular Asc and sustains intracellular Asc at higher level and for longer time.

The current positive feedback between RA and AscPNa provides additional understanding on these two vitamins (A and $\mathrm{C}$ ) and reprogramming process. In addition, the RAREs on the promoters of Gluts and Svcts and the binding sites of mesenchymal markers on the promoters of Cyp26a1 and Cyp26b1 are consistent in different types of cells. In addition, some observations were not only observed during reprogramming but also confirmed during MEF culture. Thus, the current positive feedback may not be limited to somatic reprogramming and may be applicable in other biological processes.

\section{Methods}

\section{Cell culture}

All procedures related to animal were approved by the Institutional Review Board in Guangzhou Institutes of Biomedicine and Health. Mice were normally housed in groups of four with access to food and water ad libitum. After pregnancy, mice were housed individually with access to food and water ad libitum.

MEFs were isolated from 13.5-day mouse embryos carrying the Oct4-GFP transgenic allele (Szabo et al. 2002; Ying et al. 2002). Cells were cultured in MEF medium, which contains high glucose DMEM (Gibco), 10\% FBS (Gibco), 1\% GlutaMAX (Invitrogen), and 1\% nonessential amino acids (NEAA, Invitrogen), for one passage before freezing in liquid nitrogen. MEFs were recovered from liquid nitrogen before use. MEFs were subjected to mycoplasma tests (MycoAlert ${ }^{\text {tw }}$, Lonza) to ensure they were free of mycoplasma.

\section{iPSCs generation}

The retrovirus plasmids pMX-Oct4, pMX-Klf4, pMXSox2, pMX-cMyc were delivered into Plat-E cells with by polyethyleneimine. MEF medium were used to culture Plat-E cells and viral suspensions were harvested at $48 \mathrm{~h}$ and $72 \mathrm{~h}$ after transfection.

The virus-containing medium was used to infect MEFs in 12-well plates $\left(1.5 \times 10^{4}\right.$ cells/well $)$ twice on day 0 and day 1 . Corresponding medium was then used from day 2 to induce reprogramming and replaced with the fresh medium every other day. Reprogramming efficiency was evaluated by counting the number of Oct4-GFP ${ }^{+}$ colonies.

mES medium contains high glucose DMEM, 15\% FBS, 1\% GlutaMAX, 1\% NEAA, 1\% Sodium Pyruvate (Gibco), $0.1 \mathrm{mM} \beta$-mercaptoethanol, and $1000 \mathrm{U} / \mathrm{ml}$ leukemia inhibitory factor (LIF). N2B27 medium contains DMEM/ F12 (1:1, Hyclone), 0.5\% N2 supplement (Invitrogen), 1\% B27 supplement (without Va, Invitrogen), 1\% GlutaMAX, $0.1 \mathrm{mM} \beta$-mercaptoethanol, and $1000 \mathrm{U} / \mathrm{ml} \mathrm{LIF.}$ E8 medium contains DMEM/F12, $19.4 \mathrm{mg} / \mathrm{L}$ insulin, $10.7 \mathrm{mg} / \mathrm{L}$ transferrin, and $14.7 \mathrm{ng} / \mathrm{ml}$ sodium selenium. Osmolality of all the media was adjusted to $340 \mathrm{mOsm}$ at $\mathrm{pH} 7.4$.

\section{qPCR and RNA-seq}

RNA was extracted from cells with TRIzol reagent (Thermo Fisher). RNA-seq libraries were prepared for each RNA sample using the TruSeq RNA Sample Preparation Kit v2 (RS-122-2001, Illumina). The sequencing was performed with a NextSeq 500 High Output Kit v2 (75 cycles) (FC-404-1005, Illumina) following the manufacturer's instructions. The depths of sequencing are 10 $\mathrm{M}$ pair-end reads of length 50NT. The raw data obtained from RNA-seq were tested for basic quality control with the FASTQC tool, and the filtered reads were pre-processed using the TRIMMOMATIC tool. The percentage of qualified reads was $97.37 \%$. Reads were aligned to a transcriptome index generated from the Ensembl annotations (v67), using RSEM, bowtie2, and sequencing data using GC-content normalization (Risso et al. 2011).

After normalization, genes whose expression was significantly (above 1 or below -1 when considering Log2 values) modulated by RA or AscPNa were used for GO analysis with DAVID 6.7 (https://david-d.ncifcrf.gov/) (Sherman and Lempicki 2009). 
For qPCR, total RNA was extracted with Trizol. $2 \mu \mathrm{g}$ total RNA was used to synthesize cDNA, which was then used to detect the expression of target genes by SYBR Green Master Mix ReverTra Ace qPCR-RT kit (TOYOBO). The expression of target genes was normalized to GAPDH. The primers for qPCR were listed in Table S2.

\section{Promoter activity assay}

We used the plasmid pRlenti-EGFP as backbone to detect the promoter activity. We replaced the promoter of EGFP in pRlenti-EGFP with promoters of different genes, Cyp26a1 (-3000 + 100 related to TSS), Cyp26b1 ($2000 \sim+100$ ), or three RA response elements (RAREs, CCGCTCGAGGGTGAACTTTCGGTGAACCCTACCC. TCGAGTGAACTTTCGGTGAACCCTACCCTCGA GGGTAGGGTTCACCGAAAGTTCACTCGAC) plus a mini-TK promoter. Flow cytometry analysis was performed with Accuri C6 (BD Biosciences), the average EGFP fluorescence intensity of target cells were used to indicate the promoter activity.

\section{Intracellular Asc concentration}

The intracellular Asc concentration was detected by ascorbic acid assay kit (fluorometric) (AB65346). The cells were digested with $0.25 \%$ trypsin and counted. Fluorescence was measured with a microplate reader at $\mathrm{Ex} /$ $\mathrm{Em}=535 / 590 \mathrm{~nm}$. Keep the whole process on ice and quick to avoid oxidation of Asc.

\section{Immunoblotting}

Whole cell extracts were obtained with lysis buffer $(50$ $\mathrm{mM}$ Tris pH 8.0, $150 \mathrm{mM} \mathrm{NaCl}, 10 \%$ Glycerol, $0.5 \%$ NP40, 1 mM Phenylmethanesulfonyl fluoride and freshly added protease inhibitor cocktail). Approximately $100 \mu \mathrm{g}$ of cell lysates were used for immunoblotting. Samples were loaded onto SDS-PAGE and transferred to PVDF membrane. The membrane was washed with TBST buffer and incubated with indicated antibodies (GLUT1, Abcam, ab115730; GLUT3, Abcam, ab191071; GADPH, Abcam, ab8245). Minichem Biosystem (SAGECREA TION) were used to semi-quantify the target proteins.

\section{Statistical analysis}

All experiments were repeated at least five times $(n \geq 5)$, with the exception of sequencing. The data were analyzed and compared using two-tailed t-test, one-way ANOVA, or two-way ANOVA with multiple comparisons in GraphPad Prism 7.0. Error bars and "n" represent the standard deviation (standard error if indicated) and the number of independent experiments, respectively. “*”, “***", and “*:**" represent significant differences $(P<0.05, P<0.01$, and $P<0.001$, respectively) from the indicated control groups.

\section{Supplementary information}

Supplementary information accompanies this paper at https://doi.org/10. 1186/s13619-020-00057-1.

Additional file 1 Figure S1 RA had high ability to induce MET and pluripotency. Figure S2. RA promoted neuronal differentiation during reprogramming.

Additional file 2. Supplementary Table S1 RNA-seq results in the current studies.

Additional file 3. Supplementary Table S2 The primers used in the current studies.

\section{Abbreviations}

AscPNa: 2-phospho-L-ascorbic acid trisodium salt: DHAA: L-dehydroascorbic acid; EMT: Epithelial-mesenchymal transition; ESCs: Embryonic stem cells; FBS: Fetal bovine serum; GLUTs: Glucose transporters; GO: Gene Oncology; iPSCs: Induced pluripotent stem cells; LIF: Leukemia inhibitory factor; MEFs: Mouse embryonic fibroblasts; MET: Mesenchymal-epithelial transition; NEAA: Nonessential amino acids; RA: Retinoic acid; SVCTs: sodium-dependent vitamin C transporters; Tet1: Ten-eleven translocation 1; Va: Vitamin A; Vc: Vitamin C

\section{Authors' contributions}

M.D. and Q. L. performed most of the experiments and wrote the manuscript. T. Y. and F. M. repeated experiments in Figure 5. X. L. and L.L. repeated experiments in Figure 6. C.L. and H. S. analyzed data in Figure 2. L. L, C.L., and H.Z. acquired the funding. J. S. wrote the manuscript. H. Z. supervised the studies. The authors read and approved the final manuscript.

\section{Funding}

This work was supported by the National Natural Science Foundation of China (Grant No. 31671475, U1601228, 31900699, and 81702445), the Strategic Priority Research Program of Chinese Academy of Sciences, No. XDA16010305; the Key Research Program of Frontier Sciences of Chinese Academy of Sciences, No. QYZDB-SSW-SMC031; the International Partnership Program of Chinese Academy of Sciences, No. 154144KYSB20190034; and the Key Research \& Development Program of Guangzhou Regenerative Medicine and Health Guangdong Laboratory (Grant No. 2018GZR1 10104008), and the Science and Technology Planning Project of Guangdong Province (Grant No. 2017B030314056)

\section{Availability of data and materials}

The RNA-seq datasets generated during the current study are available in Gene Expression Omnibus under accession numbers, GSE103791 and GSE142121 (https://www.ncbi.nlm.nih.gov/geo/query/acc.cgi?acc=GSE103791 and https://www.ncbi.nlm.nih.gov/geo/query/acc.cgi?acc=GSE142121).

\section{Ethics approval and consent to participate}

All procedures related to animal studies (isolate primary MEFs) were approved by the Institutional Review Board in Guangzhou Institutes of Biomedicine and Health.

\section{Competing interests}

The authors declare that they have no competing interests.

\section{Author details}

${ }^{1}$ CAS Key Laboratory of Regenerative Biology, Guangzhou Institutes of Biomedicine and Health, Chinese Academy of Sciences, \#190 Kaiyuan Ave., Science City, Guangzhou 510530, Huangpu District, China. Bioland Laboratory (Guangzhou Regenerative Medicine and Health Guangdong Laboratory), Guangzhou 510700, China. ${ }^{3}$ Guangdong Provincial Key Laboratory of Stem Cell and Regenerative Medicine, Guangzhou 510530, China. ${ }^{4}$ University of Chinese Academy of Sciences, Beijing 100049, China. ${ }^{5}$ Guangzhou Medical University, Guangzhou 511436, China. ${ }^{6}$ Institutes for Stem Cell and Regeneration, Chinese Academy of Sciences, Beijing 100101, China. 
Received: 14 April 2020 Accepted: 3 August 2020

Published online: 01 October 2020

\section{References}

Austria R, Semenzato A, Bettero A. Stability of vitamin C derivatives in solution and topical formulations. J Pharm Biomed Anal. 1997;15:795-801.

Bar-El Dadon S, Reifen R. Vitamin A and the epigenome. Crit Rev Food Sci Nutr. 2017:57:2404-11.

Chen G, Gulbranson DR, Hou Z, Bolin JM, Ruotti V, Probasco MD, et al. Chemically defined conditions for human iPSC derivation and culture. Nat Methods. 2011;8:424-9.

Chen J, Guo L, Zhang L, Wu H, Yang J, Liu H, et al. Vitamin C modulates TET1 function during somatic cell reprogramming. Nat Genet. 2013:45:1504-9.

Cui J, Gong M, He Y, Li Q, He T, Bi Y. All-trans retinoic acid inhibits proliferation, migration, invasion and induces differentiation of hepa1-6 cells through reversing EMT in vitro. Int J Oncol. 2016;48:349-57.

El Hokayem J, Weeber E, Nawaz Z. Loss of Angelman syndrome protein E6AP disrupts a novel antagonistic estrogen-retinoic acid transcriptional crosstalk in neurons. Mol Neurobiol. 2018;55:7187-200.

Esteban MA, Pei D. Vitamin C improves the quality of somatic cell reprogramming. Nat Genet. 2012;44:366-7.

Esteban MA, Wang T, Qin B, Yang J, Qin D, Cai J, et al. Vitamin C enhances the generation of mouse and human induced pluripotent stem cells. Cell Stem Cell. 2010;6:71-9.

Gao Y, Yang L, Chen L, Wang X, Wu H, Ai Z, et al. Vitamin C facilitates pluripotent stem cell maintenance by promoting pluripotency gene transcription. Biochimie. 2013;95:2107-13.

Gottesman ME, Quadro L, Blaner WS. Studies of vitamin a metabolism in mouse model systems. BioEssays. 2001;23:409-19.

Guan J, Zhang H, Wen Z, Gu Y, Cheng Y, Sun Y, et al. Retinoic acid inhibits pancreatic cancer cell migration and EMT through the downregulation of IL6 in cancer associated fibroblast cells. Cancer Lett. 2014;345:132-9.

Gudas LJ, Wagner JA. Retinoids regulate stem cell differentiation. J Cell Physiol. 2011;226:322-30

Hore TA, von Meyenn F, Ravichandran M, Bachman M, Ficz G, Oxley D, et al. Retinol and ascorbate drive erasure of epigenetic memory and enhance reprogramming to naive pluripotency by complementary mechanisms. Proc Natl Acad Sci U S A. 2016;113:12202-7.

Hou P, Li Y, Zhang X, Liu C, Guan J, Li H, et al. Pluripotent stem cells induced from mouse somatic cells by small-molecule compounds. Science. 2013;341: $651-4$.

Hu P, Tian M, Bao J, Xing G, Gu X, Gao X, et al. Retinoid regulation of the zebrafish cyp26a1 promoter. Dev Dyn. 2008;237:3798-808.

Kameyama K, Sakai C, Kondoh S, Yonemoto K, Nishiyama S, Tagawa M, et al. Inhibitory effect of magnesium L-ascorbyl-2-phosphate (VC-PMG) on melanogenesis in vitro and in vivo. J Am Acad Dermatol. 1996;34:29-33.

Li R, Liang J, Ni S, Zhou T, Qing X, Li H, et al. A mesenchymal-to-epithelial transition initiates and is required for the nuclear reprogramming of mouse fibroblasts. Cell Stem Cell. 2010;7:51-63.

Li X, Zhao Z, Zhang X, Yang S, Lin X, Yang X, et al. Klf4 reduces stemness phenotype, triggers mesenchymal-epithelial transition (MET)-like molecular changes, and prevents tumor progression in nasopharygeal carcinoma. Oncotarget. 2017;8:93924-41.

Linster CL, Van Schaftingen E. Vitamin C. Biosynthesis, recycling and degradation in mammals. FEBS J. 2007;274:1-22.

Lu J, Liu X, Zheng J, Song J, Liu Y, Ruan X, et al. Lin28A promotes IRF6-regulated aerobic glycolysis in glioma cells by stabilizing SNHG14. Cell Death Dis. 2020; $11: 447$

Masuda S, Wu J, Hishida T, Pandian GN, Sugiyama H, Izpisua Belmonte JC. Chemically induced pluripotent stem cells (CiPSCs): a transgene-free approach. J Mol Cell Biol. 2013;5:354-5.

Meneses AM, Medina RA, Kato S, Pinto M, Jaque MP, Lizama I, et al. Regulation of GLUT3 and glucose uptake by the CAMP signalling pathway in the breast cancer cell line ZR-75. J Cell Physiol. 2008;214:110-6.

Mora JR, Iwata M, von Andrian UH. Vitamin effects on the immune system: vitamins a and D take Centre stage. Nat Rev Immunol. 2008;8:685-98.

Risso D, Schwartz K, Sherlock G, Dudoit S. GC-content normalization for RNA-Seq data. BMC Bioinformatics. 2011;12:480.

Huangda W, Sherman BT, Lempicki RA. Systematic and integrative analysis of large gene lists using DAVID bioinformatics resources. Nat Protoc. 2009;4:4457.
Shi JH, Zheng B, Chen S, Ma GY, Wen JK. Retinoic acid receptor alpha mediates all-trans-retinoic acid-induced Klf4 gene expression by regulating Klf4 promoter activity in vascular smooth muscle cells. J Biol Chem. 2012;287: 10799-811.

Shu $X$, Pei D. The function and regulation of mesenchymal-to-epithelial transition in somatic cell reprogramming. Curr Opin Genet Dev. 2014;28:32-7.

Szabo PE, Hubner K, Scholer H, Mann JR. Allele-specific expression of imprinted genes in mouse migratory primordial germ cells. Mech Dev. 2002;115:15760.

Takahashi K, Yamanaka S. Induction of pluripotent stem cells from mouse embryonic and adult fibroblast cultures by defined factors. Cell. 2006;126: 663-76.

Wang T, Chen K, Zeng X, Yang J, Wu Y, Shi X, et al. The histone demethylases Jhdm1a/1b enhance somatic cell reprogramming in a vitamin-C-dependent manner. Cell Stem Cell. 2011b:9:575-87.

Wang W, Yang J, Liu H, Lu D, Chen X, Zenonos Z, et al. Rapid and efficient reprogramming of somatic cells to induced pluripotent stem cells by retinoic acid receptor gamma and liver receptor homolog 1. Proc Natl Acad Sci U S A. 2011a;108:18283-8.

Wilson JX. The physiological role of dehydroascorbic acid. FEBS Lett. 2002;527:59.

Wilson JX. Regulation of vitamin C transport. Annu Rev Nutr. 2005;25:105-25.

Wu H, Wu Y, Ai Z, Yang L, Gao Y, Du J, et al. Vitamin C enhances Nanog expression via activation of the JAK/STAT signaling pathway. Stem Cells. 2014;32:166-76.

Yang J, Wang W, Ooi J, Campos LS, Lu L, Liu P. Signalling through retinoic acid receptors is required for reprogramming of both mouse embryonic fibroblast cells and Epiblast stem cells to induced pluripotent stem cells. Stem Cells. 2015;33:1390-404.

Ying QL, Nichols J, Evans EP, Smith AG. Changing potency by spontaneous fusion. Nature. 2002:416:542-8.

Zambelli F, Pesole G, Pavesi G. Pscan: finding over-represented transcription factor binding site motifs in sequences from co-regulated or co-expressed genes. Nucleic Acids Res. 2009;37:W247-52.

Zhao Y, Zhao T, Guan J, Zhang X, Fu Y, Ye J, et al. A XEN-like state bridges somatic cells to Pluripotency during chemical reprogramming. Cell. 2015;163: 1678-91.

\section{Submit your manuscript to a SpringerOpen ${ }^{\circ}$ journal and benefit from:}

- Convenient online submission

- Rigorous peer review

- Open access: articles freely available online

- High visibility within the field

- Retaining the copyright to your article

Submit your next manuscript at $\boldsymbol{\nabla}$ springeropen.com 